\title{
Kommentare
}

\section{Obrigkeitliches Bewußtsein im Richterstand}

-Von allen Bereichen hoheitlicher Tätigkeit haben sich gerade bei der Justiz obrigkeitsstaatliche Züge am stärksten erhalten.« Derjenige, der dies geschrieben hat, gehört nicht zur Apo, es ist Rechtsanwalt Redeker, der Präsident des Deutschen Juristentags, häufiger Prozeßvertreter der Bundesregierung. ${ }^{1}$

Aber auch diesem unverfänglichen Kronzeugen werden viele Juristen, vor allem Richter, nicht glauben; sie haben es ja schwarz auf weiß, daß der Vorwurf einer obrigkeitlichen Haltung den Richter nicht treffen kann; sie verweisen auf das Grundgesetz, auf die darin erfolgte Proklamierung einer eigenständigen richterlichen Gewalt, auf die Konstitutionierung des Bundesverfassungsgerichts, auf das Vordringen von Natur- und Richterrecht in der Rechtswirklichkeit. Aber dennoch wurde das Bewußtsein der Richterschaft durch diese Vorgänge nicht berührt, sie wurden lediglich formal, aber nicht ihrem Inhalt nach aufgenommen; die Richterschaft trat $z$ war für eine Stürkung der Justiz ein, für die $•$ Entfesselung der dritten Gewalt ", wie es schon s95s der Staatsrechtslehrer Ridder ironisierte, aber sie begriff nicht, daß, was vor allem Adolf Arndt forderte', die Richter diese dritte Gewalt auch selbst politischer, demokratischer ausformen mußten, sowohl im Verhältnis zum Rechtssuchenden wie auch in dem zum Justizapparat. Intern blieb man den Strukturen des richterlichen Beamten, der strikten politischen $n$ Neutralität $\star$ und der Hierarchie des Justizapparates treu ${ }^{3}$.

Hierfür lassen sich viele Beweise bringen. Uns soll in diesem Zusammenhang die richterliche Standespolitik beschäftigen, weil sie entscheidende Hinweise auf das Selbstverständnis der Richterschaft gibt. Dabei wird es vorwiegend um den Deutschen Richterbund gehen, dem $81 \%$ der Richter und Staatsanwälte angehörent. Eine Berufsorganisation im Bereich der Arbeitnehmerschaft - letztlich ist ja auch der Richter Arbeitnehmer, er hat ja Lohnsteuer zu entrichten - bat aus der Sache heraus einen gewerkschaftlichen Charakter. Gerade wer auf der untersten Stufe der Hierarchie steht, hat ein Interesse daran, durch die Solidarität der Gleichgestellten und Gleichgesinnten gestützt und geschützt zu werden; zu Sprechern werden darum diejenigen gewählt, mit denen man sich durch das gleiche Berufsschicksal verbunden fühlt, die aber durch ihr Engagement hervorragen.

Im Richterbund jedoch wurden - und werden größtenteils noch heute - zu Sprechern der Richterschaft diejenigen gewählt, die etwas »Besseres« sind, die Dienstyorgesetzten, die also die Richter der Eingangsstufe, das Gros der Richter, beaufsichtigen, gegen sie Disziplinarstrafen verhängen, oder, als Richter höherer Gerichte, die Entscheidungen der, wie es bisweilen heißt, Unterrichter aufheben.

1 NJW 1967, 1298, Anm. 10.

- Das Bild des Richters, Müller, Karlsruhe r 956.

- Symptomatisch hierfür sind die Richterbücher von Wagner, Der Richter, Müller, Kaslsrube

I956, und Brüggemann, Die redirsprechende Gewalt, De Gruyter, Berlin I962.

4 DRiZ 1967, 33 . 
So hatten nach dem Stande vom I S. I I. I 963 von 27 Mitgliedern des Bundesvorstands 20 den Rang eines Senatspräsidenten und höher, 5 waren Direktor oder Oberlandesgerichtsrat und nur 2 Richter der Eingangsstufe. - Ein Vertreter im Vorstand kam auf 36 Richter der ersten Gruppe, auf 540 der $z$ weiten und auf \$760 - unter Mitzählung der Oberamtsrichter - der dritten'5! Dic höheren Richter sind also hunderr Mal mehr als die unteren repräsentiert. Von den Landesorganisationen des Richterbundes wurden nach dem Stande vom Sommer r 966,9 von Senatspräsidenten und höher, 3 von Direktoren oder Oberräten und keine von einem Richter der Eingangsstufe geführt ${ }^{6}$.

Der Schriftleiter der Deutschen Richterzeitung - selbst ein Bundesrichter(!) rechtfertigt das so: man finde beim Suchen nach Kollegen, dic sich zur berufsständigen Nebenarbeit bereir erklären, bei den Richtern der Eingangsstufe wenig Gegenliebe, eher hingegen bei Direktoren, die alsdann diese Arbeit nicht aus Herrsch- oder Geltungssucht, sondern aus Pflichtgefühl und Altruismus übernähmen $^{7}$. Daran ist sicher viel Wahres; aber gerade dieses Wahre indiziert hierarchische und keine demokratische Strukturen! Man sucht Schutz unter den Firtichen der Obrigkeit, bei den vertrauten Präsidenten und Vorsitzenden.

Wegen dieser Einstellung in der Richterschaft und wegen der Führung des Richterbundes durch höhere Richter, durch Honoratioren, war der Erfolg der Verbandspolitik gering. Bei Verhandlungen im politischen Raum entscheiden weniger Rang und persönliches Auftreten des Verbandsvertreters, als vielmehr das Gewicht seiner Vollmacht, inwieweit sich die Verbandsangehörigen durch ihren Vertreter repräsentiert fühlen und bereit sind, voll und ganz für ihn einzustehen. Dazu kommt, daß Honorationen keinen Zugang zu dem harten Verhandlungsstil im Krafffeld der Interessentenverbände besitzen; sie verhalten sich eben honorig und werden auch honorig behandelt und dabei mit Worten wie skein anderer Verband zeige sich wic die Richter bei Forderungen so würdig und maßvoll $\propto$ abgespeist, was arglos den Vertretenen als Erfolg weiter vermittelt wurde. Natürlich wollten sich gerade die unteren Richter, die Amtsrichter, mit derartigen verbalen Zusprüchen nicht länger begnügen, weshalb die Spirzen der Richterschaft die unhonorigste aller Fragen, die Geldfrage, gewissermaßen aus dem patriarchalischen Fürsorgegedanken heraus, immer mehr in den Mittelpunkt der Verbandspolitik stellten.

So kam es, daß ein Verband, der seiner Satzung nach zu edel und zu gut ist, das Interesse seiner Mitglieder in den Vordergrund zu stellen, der das erste seiner Ziele in der "Förderung von Gesetzgebung, Rechtspflege und Rechrswissenschaft “ sieht, in der Besoldungsfrage die einende Klammer erblidkre, die Amtsrichter und Präsidenten zusammen hieit. I 966 erschien kein Heft der Deutschen Richterzeitschrift ohne Hinweis auf Besoldungsfragen, wobei das Augustheft mit 7 Beiträgen herausragte.

Wenn somit die Besoldungsfrage einen Berufsverband, der sich als aristokratische Elite auffaßte, in den tiefsten Niederungen einer Interessentengruppierung zeigte, so gab doch gerade diese Frage das Signal für die Sammlung der gegenhierarchischen Kräfte in der Richterschaft. Hervorzuheben sind die hessischen Amtsrichter, die 1966 *völlig standeswidrig* ihren Dienstherrn auf Erhöhung der Gehälter verklagten. Bezeichnend, daß dieser Vorgang von dem damaligen Bundesjustizminister Jaeger auf dem Juristentag $\times 966$ in Essen als "Niedergang" gegeißelt wurde, bezeichnend weiter, daß die $\gg$ Mächte der Herrschaft « bei der Ju-

5 Belege DRiZ 1964, 29, 180 .

- DRiZ 1966, 35 .

7 DRiZ I967, 272. 
stizbürokratie in Hessen den klagenden Richtern wie zu Zeiten des Gottesgnadentums den Entzug der landesherrlichen Huld - der Justizminister sei wegen des Vorgehens der Richter "verprellt " und nicht in neuphorischer Stimmung* androhten ${ }^{8}$.

Mit der Kernzelle in Hessen erfolgte eine Sammlung der Richter in der Eingangsstufe gegen das Establishment im Richterbund. Anstelle eines Landgerichtspräsidenten als bisherigen Vorsizzenden des Landesverbands Hessen wurde ein Amtsrichter gewählt ${ }^{0}$. Ende I 967 konnte ein geschäftsführender Vorstand gewähit werden, in dem endlich die Richter der Eingangsstufe starik - mit 4 Personen neben gleichfalls 4 Richtern und Staatsanwälten aus der mittleren Gruppe und 2 Präsidenten - vertreten sind ${ }^{10}$. Damit ist die Unterrepräsentation der Richter der Eingangsstufe aber noch nicht beseitigt - auf knapp 2000 kommt ein Vertreter, dagegen auf 750 der mittleren Gruppe und 400 der Präsidenten -; auch ist noch ein Landgerichtspräsident Vorsitzender des Bundes; gleichwohl sind Anzeichen der Wandlung im Richterbund erkennbar. Dies ist auch schon nach außen gedrungen, wie die Stellungnahme des Vorstandes des Richterbundes nach den Studentendemonstrationen Ostern 1968 zeigte, als er die Auffordcrung aus weiten Teilen der Offentiichkeit und der etablierten Parteien, die Justiz müsse hart durchgreifen, mit dem Hinweis zurückwies, der Richter sei nicht der Bütrel der Nation.

Allerdings ist die Meinung in der Richterschaft nicht einheitlich. Auch in den unteren Instanzen wird noch vielfach, wie die Demonstrantenurteile zeigen, obrigkeitlich, wenn auch mehr unreflektiert, gedacht; der Richter sieht sich also noch als Diener des Staates, hart: als sein Büttel. Man geht wohl in der Annahme nicht fehl, daß die meisten Richter die fortschrittliche Einstellung der Reform im Richterbund nicht teilen, ja es sich hier sogar um relativ wenige handeln wird, die sich durch ihr Engagement und mit der Hilfe von - für die Reformen in der Justiz - aufgeschlossenen Teilen der öffentlichen Meinung in den Vordergrund geboxt haben.

Aber eigentlich sind selbst diese Reformer noch Traditionalisten. Letztlich gehen auch sie noch von dem hergekommenen Beamtenrichter aus, von einer dogmatisch-juristischen Vorbildung und einer - durch Auflösung der kleinen Gerichte immer stärker werdenden - Justizbürokratie. Diese Bürokratie soll allerdings, durch Mitwirkung der Richter, demokratisch ausgerichtet werden. Aber ähnlich lassen sich Modelle für die Selbstverwaltung eines Finanzamtes aufstellen. Die Reformer nehmen noch keine Kenntnis von den Prognosen der Soziologen für eine zukünftige Gesellschaft, wonach der integrative* Richtertyp keine Chancen mehr hat. Gesellschaftsrelevant wird ein zukünftiger Richter nur sein, der unbürokratisch verhandelt und entscheider, der gewissermaßen die Funktionen des Schiedsmannes in Sühnesachen und des Ombudsmannes in sich vereinigt.

Theo Rasehorn

\footnotetext{
6 Vgl. Tatbestand VerwG Frankfurt, DRiZ :966, 344.

- DRiZ $1966,389$.

$10 \mathrm{DRiZ} 1967,435$.
} 